\title{
A Cogwheel WGM Resonator Based on Spoof Surface Plasmon Polaritons
}

\author{
Peng Li, Ming Huang, Jingjing Yang, Qiannan Lu, Baojing Hu, Meixia Yang \\ School of Information Science and Engineering, Yunnan University, Kunming, China \\ Email: pli@ynu.edu.cn, huangming@ynu.edu.cn
}

How to cite this paper: Li, P., Huang, M., Yang, J.J., Lu, Q.N., Hu, B.J. and Yang, M.X. (2020) A Cogwheel WGM Resonator Based on Spoof Surface Plasmon Polaritons. Journal of Computer and Communications, 8, 102-112.

https://doi.org/10.4236/jcc.2020.812010

Received: November 17, 2020

Accepted: December 17, 2020

Published: December 24, 2020

\begin{abstract}
The optical WGM resonator plays an important role in modern physics due to its ultra-high quality factor and small volume mode. In optics, SPPs modes can effectively confine electromagnetic waves at the interface between metal and dielectric, providing extremely high sensitivity. New interesting WGM phenomena will emerge when the WGM is combined with the SPPs. In this paper, a cogwheel resonator based on spoof SPPs was designed, which can generate multi-order WGM modes. The transmission coefficients, dispersion relations and resonance modes of the WGM resonator were analyzed. The proposed resonator extends the WGM mode from optical band to microwave band, providing a new perspective for the applications of WGM mode at microwave band.
\end{abstract}

\section{Keywords}

Whispering Gallery Mode, Spoof Surface Plasmon Polaritons, Transmission Response, Dispersion Relation

\section{Introduction}

In the past two decades, the development of Whispering-Gallery-Mode (WGM) technology has made great progress in basic science and technology such as optical mechanics, physics, communications, high-performance sensors, and cavity quantum electrodynamics (QED) [1]. The capability of WGM micro-resonator to capture light can significantly enhance the interactions between light and matter, and achieve high-power energy accumulation in the resonator. The size of the resonant cavity and the refractive index of the materials will change the resonant frequency and mode distribution of WGM resonators, making WGMs widely used in the fields of biochemical sensing, optical mechanics, and nonlinear photonics. The WGM phenomenon was first discovered in the field of 
acoustics. In 1910, the British physicist Rayleigh discovered and studied the WGM phenomenon for the first time while investigating sound waves [2]. In 1969, Marcatili of Bell Laboratory extended this concept to the field of optics and proposed a ring-shaped WGM optical micro-cavity [3]. According to the different structures, WGM optical micro-cavities can be divided into many categories, including micro-sphere, micro-ring, micro-disk, micro-ring core, micro-column, micro-tubule, micro-bottle and micro-bubble, etc. Light was transmitted in the form of total reflection at the boundary of the micro-cavity, and its $Q$ value can be as high as $10^{11}$ [4]. With the improvement of micro-machining technology, micro-scale dielectric cavities with smooth surfaces can be manufactured, and the WGM phenomenon can be widely used in the optical waveband. There are three main types of common optical resonators: traditional Fabry-Perot resonators, photonic crystal micro-cavities and WGM resonators. Their differences are mainly in structure and light field distribution. Due to the limitation of the forging process of the mirror, the quality factor $\mathrm{Q}$ value of the FP micro-cavity is difficult to be very high.

The working principle of photonic crystal micro-cavity is based on the tiny defects in photonic crystal. Through the photonic crystal micro-cavity, the required resonant frequency and mode volume can be flexibly customized and applied in many fields, such as laser [5] [6] [7], sensor [8] [9], coupler [10] and other photonic devices. However, due to the limitation of precision and structure of micro-machining technology, the $\mathrm{Q}$ value of photonic crystal micro-cavity is difficult to be very high, and it also faces the problem of large coupling loss in integration. WGM micro-cavity is one of the most widely used micro-cavities at optical frequency band. Typical examples include micro-cavity disc [11] [12] [13], ring cavity [14] [15] [16], microsphere cavity [17] and micro-core cavity. The WGM optical micro-cavity has the characteristics of extremely high $\mathrm{Q}$ value (up to $10^{9}$ ), smaller mode volume, and simpler manufacturing method. It is widely used in many fields such as biomolecule detection [18] [19], extremely narrow line-width laser [20] and modulator [21] [22].

Since the discovery of WGM phenomenon, many theories have been proposed to describe the resonance of the WGM. In the early days, geometric optics and Green's function were used to investigate WGM resonance [23] [24]. Later, the Lorentz-Mie theory was developed based on the scattering phenomenon of plane electromagnetic waves on spheres and cylinders of uniform media. In 1999, Gorodetsky et al. [25] proposed a semi-analytical method to describe the near-field coupling between high intrinsic Q value with WGM and optical waveguides. Brent E et al. [26] theoretically studied the coupling between tapered fiber and microsphere cavity, and obtained the relationship between the $Q$ value of the system and the coupling loss. Combined with Lorentz-Mie theory and first-order perturbation theory, in 2003, Arnold et al. [27] presented the formula for calculating the resonant wavelength offset caused by the slight change in the refractive index of the external medium. In the same year, Smith D et al. [28] theoretically demonstrated that when multiple resonators were cascaded, the 
phenomenon of electromagnetic induced transparency will be generated by mode coupling through the spectral lines. In 2009, Pu et al. [29] realized two types of WGM laser radiation with different wavelength ranges in the same fiber, and proposed a new dual-band WGM fiber laser. In 2020, Zhang et al. [30] proposed an OAM tunable vortex micro-laser capable of supporting two degenerate WGM modes, clockwise and counterclockwise.

Optical WGM resonance is a special phenomenon formed when light propagates in a ring dielectric cavity, which requires the refractive index of the dielectric cavity to be greater than that of the external environment. The light wave coupled into the micro-cavity through the waveguide is confined in the cavity. If the phase matching condition is satisfied, a steady-state WGM resonance mode will be formed at the boundary and bounded to the equatorial plane. The WGM mode coupling phenomenon had been widely investigated at the optical band, and had been explained from the perspective of quantum optics. Unfortunately, electromagnetic wave cannot propagate on the surface of a smooth conductor at the microwave band, which greatly limits the applications of the WGM mode. At the same time, how to optimize the structure of the resonant cavity or change the mode excitation to make the coupling of WGM mode becomes easier at the microwave band is the direction of future development.

\section{Two-Dimensional Model}

A cogwheel WGM resonator based on spoof SPPs was presented and the geometric dimensions were shown in Figure 1. The materials located in the center and circumferential cogwheel shape is the ideal conductor PEC. The number of cogwheels is $N$ and the period length is $d=2 \pi R / N$. The entire structure of the model is surrounded by air.

The resonance conditions of the wheeled WGM resonator can be analyzed numerically. The magnetic field distributions outside the tooth groove can be characterized as:

$$
H_{z}^{\text {out }}=\sum_{n=-\infty}^{\infty} A_{n} H_{n}^{(1)}\left(k_{0} \rho\right) e^{\text {in } \varphi}
$$

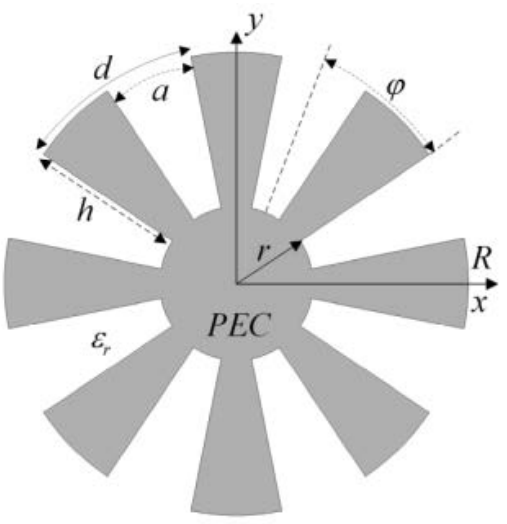

Figure 1. Structure diagram of wheeled WGM resonator. 
where $A_{n}$ is a constant, $H_{n}^{(1)}$ is the first Hankel function of $n$ order, $k_{0}$ is the wave vector in the free space, and $(\rho, \varphi)$ denotes the cylindrical coordinate system. The geometric dimensions of the cogwheel should be in the sub-wavelength range, which satisfies $\lambda_{0}>d$ ( $\lambda_{0}$ is the wavelength of the incident wave). Therefore, when the electromagnetic modes in the groove are expanded, only the basic radiation waveguide mode needs to be considered, namely:

$$
H_{z}^{g}=B^{+} H_{0}^{(1)}\left(k_{0} n_{g} \rho\right)+B^{-} H_{0}^{(2)}\left(k_{0} n_{g} \rho\right)
$$

where $B^{+}$and $B^{-}$are the coefficients of the outward radiation mode and the inward propagation mode. The phenomenon with WGM resonance can occur for any $n$ order in Equations (1) and (2) when $H_{z}$ satisfies boundary conditions. Therefore, the following equations can be obtained:

$$
\begin{gathered}
S_{n}^{2} \frac{H_{n}^{(1)}\left(k_{0} R\right)}{H_{n}^{(1) \prime}\left(k_{0} R\right)} \frac{f}{g}=n_{g} \\
f=J_{1}\left(k_{0} n_{g} r\right) Y_{1}\left(k_{0} n_{g} R\right)-J_{1}\left(k_{0} n_{g} R\right) Y_{1}\left(k_{0} n_{g} r\right) \\
g=J_{0}\left(k_{0} n_{g} R\right) Y_{1}\left(k_{0} n_{g} r\right)-J_{1}\left(k_{0} n_{g} r\right) Y_{1}\left(k_{0} n_{g} R\right)
\end{gathered}
$$

where $J_{0}$ and $J_{1}$ are the zero-order and first-order Bessel functions with the first kind, $Y_{0}$ and $Y_{1}$ are the zero-order and first-order Bessel functions with the second kind, $n$ is the number of lobe modes ( $n=1$ stands for two lobes). $S_{n}$ denotes the overlap integral between the $N$-th order plane wave and the basic TE mode, which can be expressed as $S_{n}=\sqrt{a / d} \arcsin (n a / 2 R)$. Since all the dimensions of the groove unit on the circumference are included in the calculation of $S_{n}$, and $S_{n}$ is approximated as $S_{n} \approx \sqrt{a / d}$.

According to the theory of electromagnetic metamaterials, the corrugated metal structure in sub-wavelength range can be equivalent to a metamaterial with a thickness of $h$ at the optical band. As well as at microwave band, our group [31] had proposed a dielectric disc to generate multi-order WGM modes, and the disk was excited by aluminum $(\varepsilon=10)$ as a waveguide. When the dielectric disk was close to the aluminum waveguide, spoof SPP wave was generated on the corrugated surface of the disk due to resonance, forming multi-order WGM modes. The radius of the resonant disk satisfied [32]:

$$
R=0.89 \pi c / \omega_{a}
$$

where $c$ is the speed of light in vacuum; $\omega_{a}$ is the plasmon-like frequency depending on the size of the wheeled resonator. When $R=10 \mathrm{~mm}, \omega_{a}$ can be calculated as $\omega_{a}=8.3881 \times 10^{10} \mathrm{rad} / \mathrm{m}$, and then $f=13.35 \mathrm{GHz}$. In Figure 1, the material of the wheeled disc is PEC, and the permittivity satisfies dispersion relationship of Drude model $\varepsilon_{p}(\omega)=1-\omega_{p}^{2} / \omega^{2}$, and $\omega_{p}=\sqrt{2} \omega_{a}$ is the plasmon frequency. With the variation of incident wave frequency, $\omega$ will be changed, and so does the $\varepsilon_{p}$ of wheeled resonator.

By placing a rectangular metal strip under the cogwheel (shown in Figure 1), a WGM resonator with spoof SPPs is designed. The dimensions of the resonator 
are as bellow: outer radius $R=10 \mathrm{~mm}$, inner radius $r=5 \mathrm{~mm}$, number of cogwheel $N=20$, and distance between resonator and micro-strip $g=1.5 \mathrm{~mm}$. For comparison, we simulated the magnetic field distributions $\left|H_{z}\right|$ of two structures, resonator with spoof SPPs and metamaterial resonator disk (both of infinite thickness) in COMSOL Multiphysics software, as shown in Figure 2.

It can be seen from Figure 2 that the wheeled resonator with spoof SPPs, as the metamaterial disk, can generate 5th and 6th order WGM modes at the interface between the two dielectrics. The frequencies of the 5th and the 6th order WGM modes generated by wheeled resonator are $13.14 \mathrm{GHz}$ and $13.89 \mathrm{GHz}$, while the corresponding WGM modes generated by the metamaterial disk are 12.77 $\mathrm{GHz}$ and $13.09 \mathrm{GHz}$. In addition, the same magnetic field distributions appear at the interface of the two dielectrics in these two structures. It shows that the wheeled resonator composed of corrugated grooves can be equivalent to a metamaterial disk, and both of them can generate multi-order WGM modes. Moreover, a maximum of 9-order WGM modes can occur near the frequency $f=13.35 \mathrm{GHz}$ for the proposed geometric size.

\section{Three-Dimensional Model}

The wheeled resonator with spoof SPPs discussed above was a $2 \mathrm{D}$ planar structure in COMSOL Multiphysics software, which can be equivalent to the infinite transverse thickness of the model. As a practical device, the resonator has a certain size and thickness. Therefore, we proposed a 3D WGM resonator here with a thickness of $0.035 \mathrm{~mm}$ in CST software, as shown in Figure 3(a). The entire
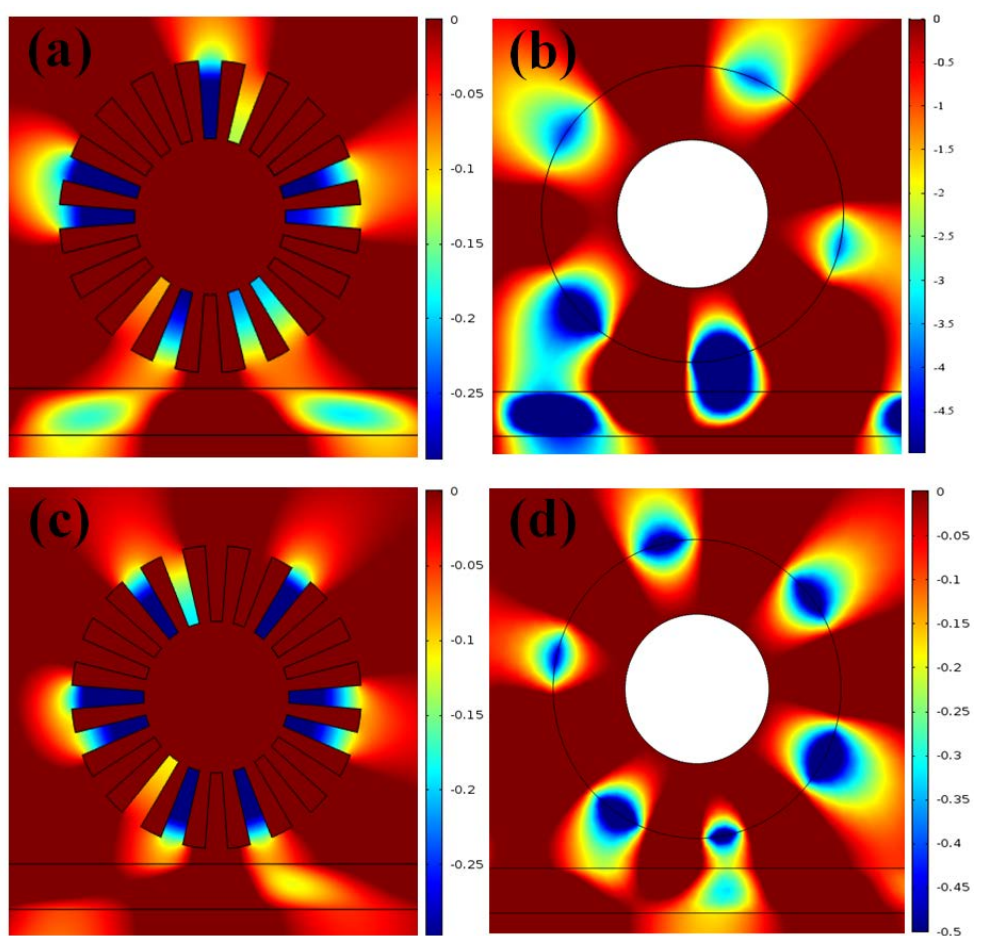

Figure 2. $\left|H_{z}\right|$ Distributions of 5th and 6th order WGM modes of two structures. 

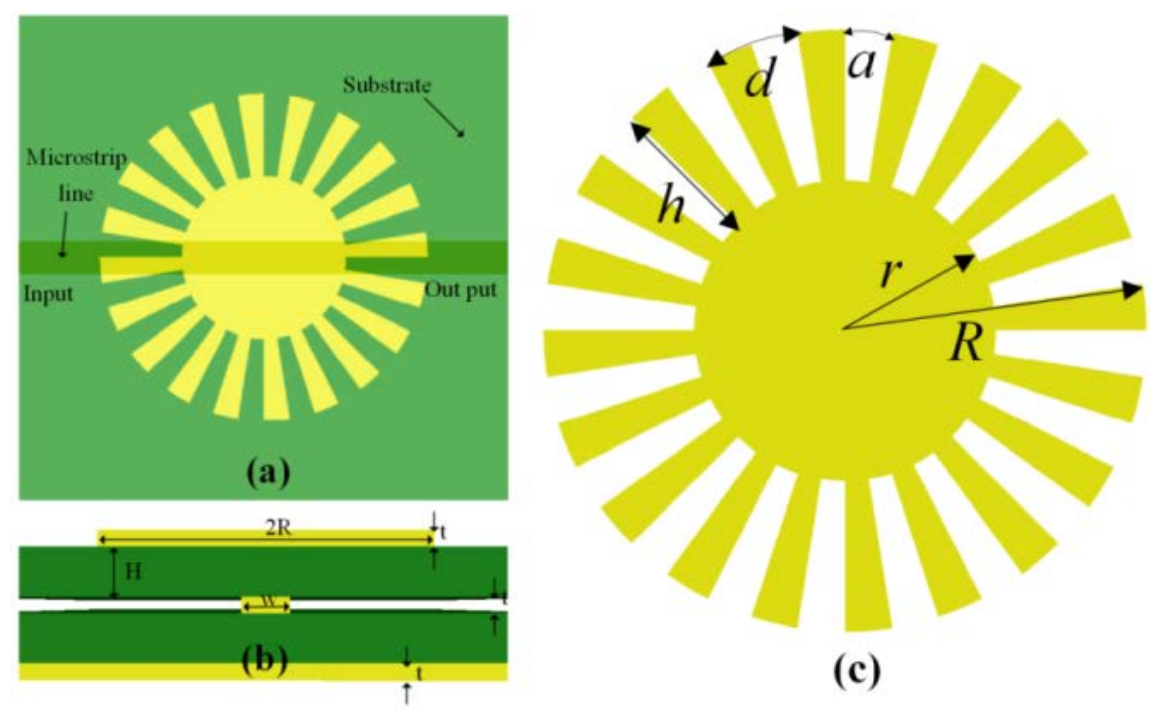

(c)

Figure 3. Structure of WGM resonator with spoof SPPs.

structure of the resonator is divided into five layers. The structure is composed of WGM resonator, dielectric substrate, excitation micro-strip, dielectric substrate and metal ground in order from top to bottom, as shown in Figure 3(b). The excitation signal is input from the left port of the rectangle micro-strip (the third layer) and output from the right port. The dimensions of the resonator are as follows: the thickness of metal layer $t=0.035 \mathrm{~mm}$, the width of micro-strip $w=2.28 \mathrm{~mm}$, the thickness of substrate $H=1 \mathrm{~mm}$. The length and width of the dielectric substrate are both $30 \mathrm{~mm}$. The surface wheeled structure on the first-layer of WGM resonator is shown in Figure 3(c). The dimensions are as bellows: $r=5 \mathrm{~mm}, R=10 \mathrm{~mm}, d=3.14 \mathrm{~mm}, a=d / 2=1.57 \mathrm{~mm}, h=5 \mathrm{~mm}$ and $n=20$.

The EM wave radiates to the outside space when it propagates through the metal micros-trip (the third layer). The EM wave is coupled through the dielectric substrate into the WGM resonator and propagating on the surface. Since the cavity of resonator is a periodic corrugated structure, the wheeled resonator will generate a WGM mode when the resonance condition is satisfied. The S21 transmission response of the presented WGM resonator is calculated in the CST software, as shown in Figure 4(a). In the range of $0-10 \mathrm{GHz}$, a total of 8 resonance peaks is appeared (marked in Figure 4(a)), which means that there are 8 resonance frequencies in the WGM resonator. To represent the WGM modes more intuitively, electric field distributions $\left(E_{z}\right)$ at a distance of $0.1 \mathrm{~mm}$ above the resonator are plotted, corresponding to the 8 resonance peaks of S21, as shown in Figures 4(b)-(i). It can be seen that $1-8$ orders WGM resonance modes are generated at these 8 resonance frequency points. The resonator generates dipole (Mode 1), quadrupole (Mode 2), hexapole (Mode 3), octopole (Mode 4), decapole (Mode 5), dodecapole (Mode 6), fourteen-pole (Mode 7), and sixteen-pole (Mode 8) WGM resonance modes respectively. 

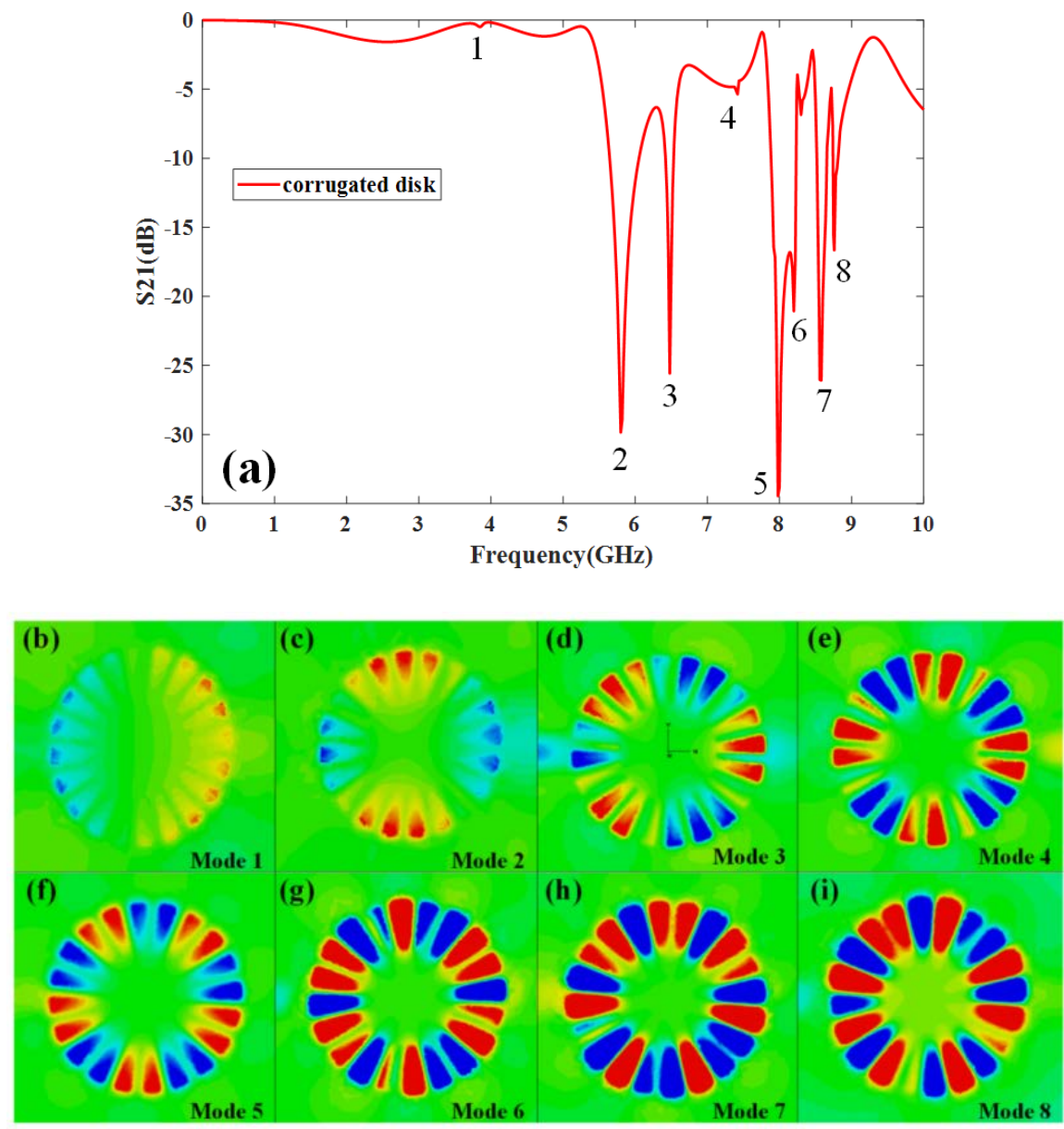

Figure 4. (a) S21 transmission response of the WGM resonator; ((b)-(i)) surface electric field distributions.

The $\mathrm{Q}$ value of the resonator represents the resonant energy of the WGM mode at the resonance point. The $\mathrm{Q}$ factor is calculated through $Q=f_{0} / \Delta f=f_{0} /\left(f_{2}-f_{1}\right)$. Here, $f_{0}$ and $\Delta f$ are the center frequency and $3 \mathrm{~dB}$ bandwidth of the spectrum. The $\mathrm{Q}$ values for 8 resonance frequencies (Figure $4(\mathrm{a})$ ) of this WGM resonator are calculated, as listed in Table 1. It is seen from Table 1 that the $\mathrm{Q}$ factor of Mode 8 reaches the maximum value of 521, where the peak of the waveform is the sharpest. The Q value of Mode 3 is in the second place, with a value of 444, and its resonance peak is also sharper. Mode 5 has the maximum attenuation in S21 transmission response, whose S21 value is $34.45 \mathrm{~dB}$ and $\mathrm{Q}$ value is 254 . Although the $\mathrm{Q}$ value of the 5 th order WGM mode is not the maximum, the S21 value is the minimum. Furthermore, among all modes, the 5th order has the most EM waves that coupled into WGM resonator. In the electric field distributions, the 5th order WGM mode in Figure 4(f) is also the clearest, which is consistent with the S21 result.

Using Eigen Solver, the dispersion relations of proposed resonator were simulated and calculated, as shown in Figure 5. The inset shows a corrugated unit structure of the resonator, whose dimension parameters are the same as those in 


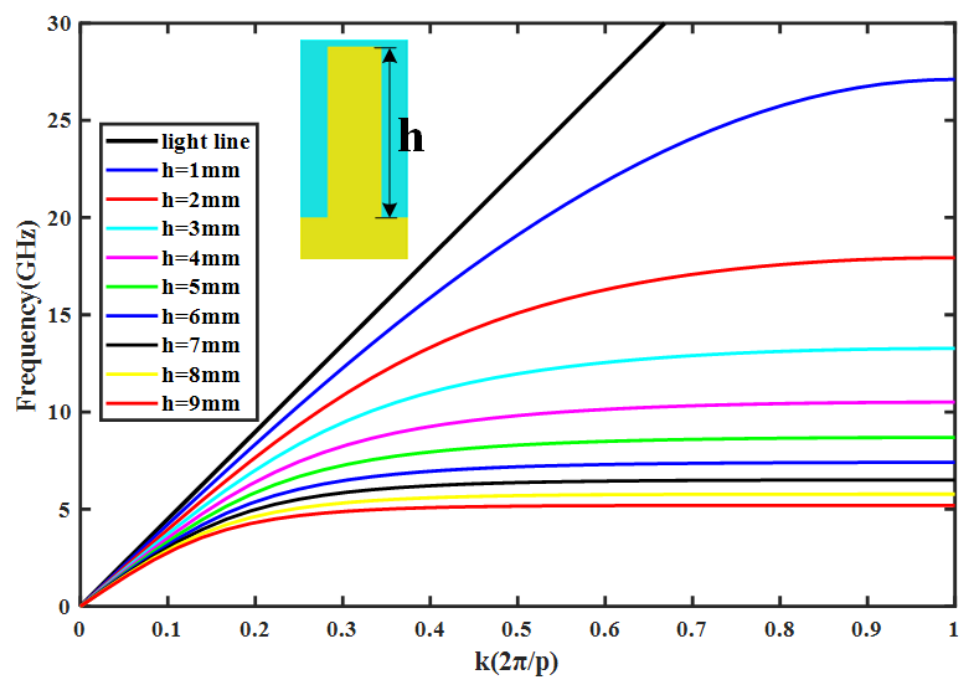

Figure 5. Dispersion relations of a unit cell with various depths of $h$.

Table 1. Q values corresponding to the multi-order WGM resonance modes.

\begin{tabular}{ccccccccc}
\hline number of modes & 1 & 2 & 3 & 4 & 5 & 6 & 7 & 8 \\
\hline Frequencies $(\mathrm{GHz})$ & 3.84 & 5.81 & 6.48 & 7.42 & 7.98 & 8.21 & 8.58 & 8.76 \\
Q values & 16 & 135 & 444 & 12 & 254 & 229 & 236 & 521 \\
\hline
\end{tabular}

Figure 3. According to the dispersion theory, the asymptotic frequency will be changed with the variation of the groove depth. As can be seen from Figure 5, the resonant frequency gradually decreases from $27.11 \mathrm{GHz}$ to $5.19 \mathrm{GHz}$ when the depth of the groove is in the range of $1-9 \mathrm{~mm}$. The results indicate that the deeper the grooves, the smaller the asymptotic frequency. It means that the SPP wave will become more confined on the surface of WGM resonator. The asymptotic frequency is $8.69 \mathrm{GHz}$ at $\mathrm{h}=5 \mathrm{~mm}$, which is consistent with the maximum value of $8.76 \mathrm{GHz}$ corresponding to the 8th order WGM mode in Figure 4. It indicates that EM waves with frequencies above $8.76 \mathrm{GHz}$ will be cut off, that is, there is no resonant mode higher than 8th order in the WGM resonator.

\section{Conclusion}

In summary, a WGM resonator with spoof SPPs is designed and demonstrated. The proposed resonator is composed of a 5-layer structure, and the characteristics of the WGM mode are discussed. Simulation results show that strongly confined SPP wave can be supported and $1-8$ orders WGM modes can be generated by the proposed resonator with wheeled structure. Furthermore, the electromagnetic response can be flexibly engineered by choosing the depth and radius of the resonator. Finally, the dispersion relation is calculated to illustrate the principle of the WGM resonator. Excellent resonant performance indicates that the WGM resonator can be used as sensors in the mid-IR, THz, and microwave regimes with many potential applications. 


\section{Acknowledgements}

Our work was funded by the National Natural Science Foundation of China (Grant Nos. 11564044, 61863035, 61963037) and Scientific Research Fund of Yunnan Education Department (Grant No. 2020J0005).

\section{Conflicts of Interest}

The authors declare no conflicts of interest regarding the publication of this paper.

\section{References}

[1] Jiang, X. and Yang, L. (2020) Optothermal Dynamics in Whispering Gallery Microresonators. Light. Science \& Applications, 9, 24.

https://doi.org/10.1038/s41377-019-0239-6

[2] Rayleigh, L. (1910) The Problem of the Whispering Gallery. Philosophical Magazine, 20, 1001-1004. https://doi.org/10.1080/14786441008636993

[3] Marcatili, E.A.J. (1969) Bends in Optical Dielectric Guides. Bell System Technical Journal, 48, 2103-2133. https://doi.org/10.1002/j.1538-7305.1969.tb01167.x

[4] Vahala, K.J. (2003) Optical Microcavities. Nature, 424, 839-846. https://doi.org/10.1038/nature01939

[5] Painter, O. (1999) Two-Dimensional Photonic Band-Gap Defect Mode Laser. Science, 284, 1819-1821. https://doi.org/10.1126/science.284.5421.1819

[6] Heo, J., Guo, W. and Bhattacharya, P. (2011) Monolithic Single GaN Nanowire Laser with Photonic Crystal Microcavity on Silicon. Applied Physics Letters, 98, 021110. https://doi.org/10.1063/1.3540688

[7] Ellis, B., Mayer, M.A., Shambat, G., et al. (2011) Ultralow-Threshold Electrically Pumped Quantum-Dot Photonic-Crystal Nanocavity Laser. Nature Photonics, 5, 297-300. https://doi.org/10.1038/nphoton.2011.51

[8] Chakravarty, S., Zou, Y., Lai, W.C., et al. (2012) Slow Light Engineering for High Q High Sensitivity Photonic Crystal Microcavity Biosensors in Silicon. Biosensors \& Bioelectronics, 38, 170-176. https://doi.org/10.1016/j.bios.2012.05.016

[9] Heiniger, A.T., Miller, B.L. and Fauchet, P.M. (2015) Numerical Study of Sensitivity Enhancement in a Photonic Crystal Microcavity Biosensor Due to Optical Forces. Optics Express, 23, 25072. https://doi.org/10.1364/OE.23.025072

[10] McGurn, A.R. (2002) Photonic Crystal Circuits: Localized Modes and Waveguide Couplers. Physical Review B, 65, 075406. https://doi.org/10.1103/PhysRevB.65.075406

[11] Schmidt, C., Chipouline, A.K., Sebier, T., et al. (2009) Observation of Optical Coupling in Microdisk Resonators. Physical Review A, 80, 043841. https://doi.org/10.1103/PhysRevA.80.043841

[12] Perahia, R., Alegre, T.P.M., Safavi-Naeini, A.H., et al. (2009) Surface-Plasmon Mode Hybridization in Sub-Wavelength Microdisk Lasers. Applied Physics Letters, 95, 20. https://doi.org/10.1063/1.3266843

[13] Diallo, S., Lin, G.P. and Chembo, Y.K. (2015) Giant Thermo-Optical Relaxation Oscillations in Millimeter-Size Whispering Gallery Mode Disk Resonators. Optics Letters, 40, 3834-3837. https://doi.org/10.1364/OL.40.003834

[14] Chao, C.Y., Fung, W. and Guo, L.J. (2006) Polymer Microring Resonators for Bio- 
chemical Sensing Applications. IEEE Journal of Selected Topics in Quantum Electronics, 12, 134-142. https://doi.org/10.1109/JSTQE.2005.862945

[15] Nawrocka, M.S., Liu, T., Wang, X., et al. (2006) Tunable Silicon Microring Resonator with Wide Free Spectral Range. Applied Physics Letters, 89, 071110.

https://doi.org/10.1063/1.2337162

[16] Wang, T., Liu, F., Wang, J., et al. (2009) Pulse Delay and Advancement in SOI Microring Resonators with Mutual Mode Coupling. Journal of Lightwave Technology, 27, 4734-4743. https://doi.org/10.1109/JLT.2009.2026590

[17] Collot, L., Lefèvre-Seguin, V., Brune, M., et al. (2007) Very High-Q Whispering-Gallery Mode Resonances Observed on Fused Silica Microspheres. EPL (Europhysics Letters), 23, 327. https://doi.org/10.1209/0295-5075/23/5/005

[18] Vollmer, F., Arnold, S. and Keng, D. (2008) Single Virus Detection from the Reactive Shift of a Whispering-Gallery Mode. Proceedings of the National Academy of Sciences of the USA, 105, 20701-20704. https://doi.org/10.1073/pnas.0808988106

[19] Santiago-Cordoba, M.A., Boriskina, S.V., Vollmer, F., et al. (2011) Nanoparticle-Based Protein Detection by Optical Shift of a Resonant Microcavity. Applied Physics Letters, 99, 073701. https://doi.org/10.1063/1.3599706

[20] Yang, L., Lu, T., Carmon, T., et al. (2007) A 4-Hz Fundamental Linewidth on-Chip Microlaser. Conference on Lasers and Electro-Optics (Optical Society of America), 6-11 May, 2007. https://doi.org/10.1109/CLEO.2007.4452623

[21] Yuce, E., Gurlu, O. and Serpenguzel, A. (2009) Optical Modulation with Silicon Microspheres. IEEE Photonics Technology Letters, 21, 1481-1483.

https://doi.org/10.1109/LPT.2009.2027891

[22] Savchenkov, A.A., Liang, W., Matsko, A.B., et al. (2009) Tunable Optical Single-Sideband Modulator with Complete Sideband Suppression. Optics Letters, 34, 1300-1302. https://doi.org/10.1364/OL.34.001300

[23] Wasylkiwskyj, W. (1975) Diffraction by a Concave Perfectly Conducting Circular Cylinder. IEEE Transactions on Antennas \& Propagation, 23, 480-492. https://doi.org/10.1109/TAP.1975.1141109

[24] Ishihara, T., Felsen, L.B. and Green, A. (1978) High-Frequency Fields Excited by a Line Source Located on a Perfectly Conducting Concave Cylindrical Surface. IEEE Transactions on Antennas and Propagation, 26, 757-767. https://doi.org/10.1109/TAP.1978.1141940

[25] Gorodetsky, M.L. and Ilchenko, V.S. (1999) Optical Microsphere Resonators: Optimal Coupling to High-Q Whispering-Gallery Modes. Journal of the Optical Society of America B, 16, 147-154. https://doi.org/10.1364/JOSAB.16.000147

[26] Little, B.E., Laine, J.P., Haus, H.A., et al. (1999) Analytic Theory of Coupling from Tapered Fibers and Half-Blocks into Microsphere Resonators. Journal of Lightwave Technology, 17, 704-715. https://doi.org/10.1109/50.754802

[27] Teraoka, I., Arnold, S. and Vollmer. F. (2003) Perturbation Approach to Resonance Shifts of Whispering-Gallery Modes in a Dielectric Microsphere as a Probe of a Surrounding Medium. Journal of the Optical Society of America B, 20, 1937-1946. https://doi.org/10.1364/JOSAB.20.001937

[28] Smith, D.D., Chang, H., Fuller, K.A., et al. (2003) Coupled-Resonator-Induced Transparency. Physical Review A, 69, 666-670. https://doi.org/10.1103/PhysRevA.69.063804

[29] Pu, X.-Y., Bai, R., Xiang, W.-L., Du, F. and Jiang, N. (2008) Two-Wavelength-Range Whispering-Gallery-Mode Fiber Laser Pumped by Evanescent Wave. Acta Physica 
Sinica, 58, 3923-3928.

[30] Zhang, Z.F., Qiao, X.D., Midya, B., et al. (2020) Tunable Topological Charge Vortex Microlaser. Science, 368, 760-763. https://doi.org/10.1126/science.aba8996

[31] Yang, J.J., Huang, M., Dai, X.Z., et al. (2013) A Spoof Surface WGM Sensor Based on a Textured PEC Cylinder. Epl, 103, 44001. https://doi.org/10.1209/0295-5075/103/44001

[32] Pors, A., Moreno, E., Martin-Moreno, L., et al. (2012) Localized Spoof Plasmons Srise While Texturing Closed Surfaces. Physical Review Letters, 108, 223905. https://doi.org/10.1103/PhysRevLett.108.223905 